\title{
GENERAL, EPIDEMIOLOGICAL PARAMETERS AND IMMUNIZATION COVERAGE OF CHILDREN SUFFERING FROM MORBILLI IN CENTRAL KOSOVO AND METOHIJA
}

\author{
Vanja Ničković ${ }^{1}$, Aleksandar Ranković2, ${ }^{2}$, Ljiljana Šulović3, Snežana Danić-Filipović3, \\ Snežana Marković-Jovanović3 ${ }^{3}$ Zorica Vujnović-Živkovićs ${ }^{3}$ Jadranka Mitić3, \\ Hristina Kocić, ${ }^{4}$ Ilija Kocićs, Marko Ristić ${ }^{6}$
}

(Vol 58, No 4, December, 2019)

\author{
${ }^{1}$ Clinical-Hospital Center, Priština, Gračanica, Serbia \\ ${ }^{2}$ Clinic of Infective Desease Niš, Serbia \\ ${ }^{3}$ University of Priština, Faculty of Medicine, Kosovska Mitrovica, Serbia \\ ${ }^{4}$ Clinical-Hospital Center, Niš, Serbia \\ ${ }^{5}$ University of Niš, Faculty of Medicine, Niš, Serbia \\ ${ }^{6}$ Phd Student, University of Niš, Faculty of Medicine, Niš, Serbia
}

In the paper titled "GENERAL, EPIDEMIOLOGICAL PARAMETERS AND IMMUNIZATION COVERAGE OF CHILDREN SUFFERING FROM MORBILLI IN CENTRAL KOSOVO AND METOHIJA" by Vanja Ničković, Aleksandar Ranković, Ljiljana Šulović, Snežana Danić-Filipović, Snežana MarkovićJovanović, Zorica Vujnović-Živković, Jadranka Mitić, Hristina Kocić, Ilija Kocić, Marko Ristić, published in AMM journal in 2019, number 58 (4), there occurred a technical error on the page 3241 , with published wrong list of authors for the paper. We hereby apologize to the authors and readers. With authors' approval, we are now publishing the correct summary. 


\title{
OPŠTI I EPIDEMIOLOŠKI PARAMETRI I IMUNIZACIJA DECE OBOLELE OD MORBILA NA CENTRALNOM KOSOVU I METOHIJI
}

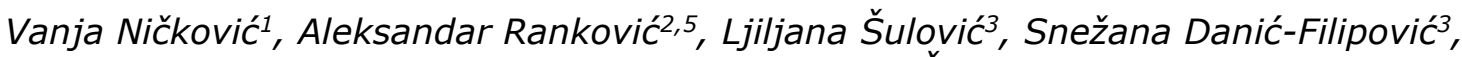 \\ Snežana Marković-Jovanović3, Zorica Vujnović-Živkovićs , Jadranka Mitić3, \\ Hristina Kocić4 ${ }^{4}$ Ilija Kocićs, Marko Ristić6
}

(Vol 58, No 4, Decembar, 2019)

${ }^{1}$ Kliničko bolnički centar Priština, Gračanica, Srbija
${ }^{2}$ Infektivna klinika Niš, Niš, Srbija
${ }^{3}$ Univerzitet u Prištini, Medicinski fakultet, Kosovska Mitrovica, Srbija
${ }^{4}$ Klinički centar Niš, Niš, Srbija
${ }^{5}$ Univerzitet u Nišu, Medicinski fakultet, Niš, Srbija
${ }^{6}$ Student poslediplomskih studija, Univerzitet u Nišu, Medicinski fakultet, Niš, Srbija

U radu "OPŠTI I EPIDEMIOLOŠKI PARAMETRI I IMUNIZACIJA DECE OBOLELE OD MORBILA NA CENTRALNOM KOSOVU I METOHIJI" autora Vanja Ničković, Aleksandar Ranković, Ljiljana Šulović, Snežana Danić-Filipović, Snežana Marković-Jovanović, Zorica Vujnović-Živković, Jadranka Mitić, Hristina Kocić, Ilija Kocić, Marko Ristić, objavljenom u časopisu AMM za 2019. godinu broj 58 (4) na stranama od 32-41, došlo je do tehničke greške, pri kojoj je odštampan pogrešan spisak autora za ovaj rad. Ovom prilikom se izvinjavamo autorima i čitaocima. Uz saglasnost autora, u ovom broju objavljujemo ispravan spisak autora.

Acta Medica Medianae 2020;59(2):132-133.

This work is licensed under a Creative Commons Attribution 4.0 International (CC BY 4.0) Licence 